\title{
Nonlinear Optical Mode Coupling by Ionization in an Ar-Filled Capillary with High-Power Short-Pulse Excitation
}

\author{
P. Horak ${ }^{1}$, R. T. Chapman ${ }^{2}$, F. Poletti ${ }^{1}$, J. G. Frey ${ }^{2}$, W. S. Brocklesby ${ }^{1}$ \\ 1. Optoelectronics Research Centre, University of Southampton, Southampton SO17 1BJ, United Kingdom \\ 2. School of Chemistry, University of Southampton, Southampton SO17 1BJ, United Kingdom
}

High-power ultrashort laser pulses at near-infrared wavelengths propagating in gas-filled capillaries can form a compact source of XUV/soft X-ray radiation by high-harmonic generation (HHG) [1]. Maximization of the frequency conversion efficiency requires a detailed understanding of the atomic interaction mechanism as well as the propagation properties of both the near-infrared pump in the presence of a partially ionized gas and of the generated XUV. Here we focus on the numerical simulation of pulse propagation in a parameter regime dominated by plasma effects and by the nonlinear properties of ionization, and compare the results with experimental observations.

The experiment [1] was performed with an Ar-filled capillary of $150 \mu \mathrm{m}$ hole diameter and $40 \mathrm{fs}$ pump pulses at $780 \mathrm{~nm}$ wavelength. At this wavelength the capillary supports a large number of optical modes which are nonlinearly coupled by the ionization processes and therefore should be included in a numerical model. However, because of mode symmetries for an optimized $\mathrm{EH}_{11}$ pump mode, coupling is predominantly to other $\mathrm{EH}_{1 \mathrm{n}}$ modes [2]. We performed simulations including the lowest 20 modes based on a generalized multimode nonlinear Schrödinger equation [2,3] with the ionization effects modeled according to the Keldysh theory [4].

(a)

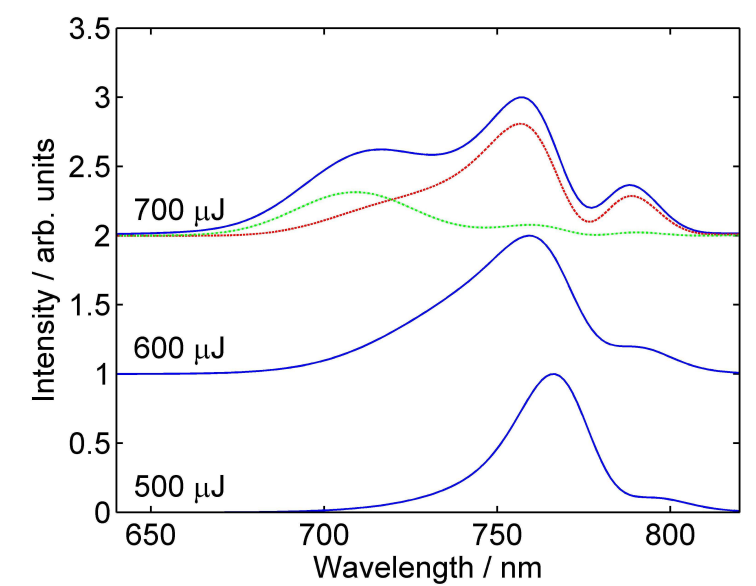

(b)

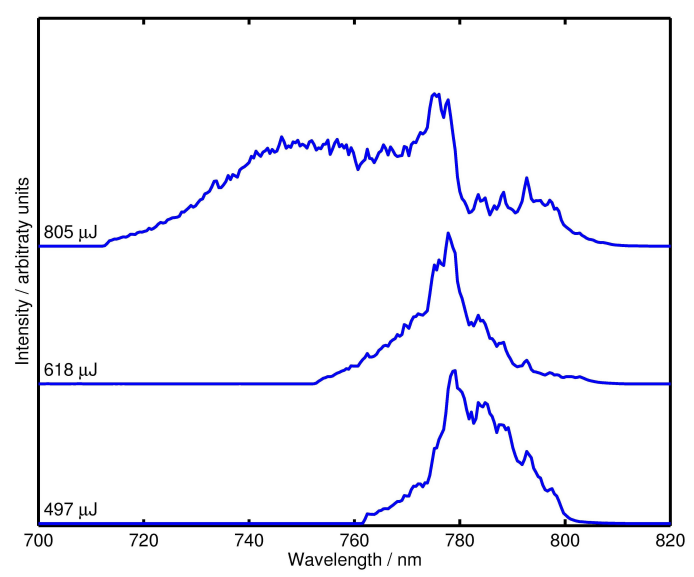

Fig. 1. (a) Simulated and (b) experimental output spectra after short-pulse propagation in a $7 \mathrm{~cm}$ long Ar-filled capillary at different input pulse energies. The modal content for the simulated spectrum at $700 \mu \mathrm{J}$ input is shown for the fundamental mode (red) and the first higher order mode (green).

Fig. 1(a) shows numerically obtained spectra at the output of a $7 \mathrm{~cm}$ capillary for various pulse energies. For increasing energies, the spectra develop a multiple peak structure comprising one peak near the initial pump wavelength, a broad blue-shifted shoulder and a third, smaller peak at slightly red-shifted wavelengths. These simulation results are closely matched by corresponding experiments, Fig. 1(b). Analyzing the modal content of the simulated output in more detail, we find that the blue-shifted peak is predominantly found in the higher order modes, as indicated by the green curve in Fig. 1(a). Initial experimental efforts to spatially resolve the output spectra confirm this prediction. Further simulations at varying Ar pressure also agree well with the experiments.

These initial results give confidence in the accuracy of the numerical simulations. The model also predicts the full radial and longitudinal distribution of the plasma density as well as the temporal dynamics, and thus it should prove useful to gain a deeper understanding of the interplay between different linear and nonlinear processes leading to XUV generation by HHG. Ultimately, this should allow us to optimize the conversion efficiency.

\section{References}

[1] M. Praeger et al., "Spatially resolved soft X-ray spectrometry from single-image diffraction," Nature Phys. 3, 176 (2007).

[2] F. Poletti and P. Horak, "Description of ultrashort pulse propagation in multimode optical fibers," J. Opt. Soc. Am. B 25,1645 (2008).

[3] C. Courtois, A. Couairon, B. Cros, J. R. Marques, G. Matthieussent, "Propagation of intense ultrashort laser pulses in a plasma filled capillary tube: Simulations and experiments," Phys. Plasmas 8, 3445 (2001).

[4] V. S. Popov, "Tunnel and multiphoton ionization of atoms and ions in a strong laser field (Keldysh theory)," Phys. Uspekhi 47, 855 (2004). 